\title{
Surface-field-induced microstructures of asymmetric diblock copolymer nanoparticles
}

\author{
Shiben $\mathrm{Li}^{1}$, Meijiao Liu ${ }^{1}$, Yongyun $\mathrm{Ji}^{1}$, Linxi $\mathrm{Zhang}^{1}$ and Haojun Liang ${ }^{2}$ \\ The microstructures of asymmetric diblock copolymer nanoparticles were investigated using real-space self-consistent field \\ theory. The nanoparticle boundary provides a spherical confinement in which the highly deformable copolymers are forced into \\ a closed and crowded space. The surface-field-induced effects on the microstructures were examined systematically, and a rich \\ variety of novel structures, such as mixtures of distorted cylinders and spherical lamellae, was observed in the copolymer \\ nanoparticles. Investigations of the free energies indicate that the surface-field-induced structural transitions are of the first \\ order. The formation mechanism of the confinement-induced structures was reasonably elucidated, based on the interactions \\ between the polymer chains and confinement boundaries, and on the spherical symmetry from confinements. Our theoretical \\ results are in good agreement with available simulation and experimental observations.
}

Polymer Journal (2011) 43, 606-612; doi:10.1038/pj.2011.35; published online 27 April 2011

Keywords: diblock copolymer; microstructure; nanoparticle; surface field

\section{INTRODUCTION}

Block copolymer nanoparticles have a broad range of applications as advanced functional materials in the optical, electronic, biological and medical fields. ${ }^{1-5}$ For example, multilayered diblock copolymer nanoparticles can be considered not only as spherical dielectric resonators ${ }^{3}$ but also as high-density three-dimensional optical data storage ${ }^{5}$ because of their inner microstructures, which originate from phase separations in a confined environment. These inner microstructures of polymer nanoparticles are intimately related to their spherical confinement boundaries, which are characterized by the confinement size and surface field, and dominate the chemical and physical properties of polymer nanoparticles.

It is generally recognized that surface fields with special symmetries are crucial to obtaining surface-induced microstructures of diblock copolymers. A large number of studies have contributed to the confinement-induced effects on the microstructures of diblock copolymers confined in thin films and cylindrical nanopores. ${ }^{6-20}$ The results of these studies indicate that cylindrical nanopores engender distinct confinement-induced effects on the microstructures compared with those of thin films because of the different symmetries of their confinement surfaces. When the polymer chains are confined in a spherical environment provided by the nanoparticle boundaries, they may show unique characteristics. Indeed, a number of novel microstructures have been observed in lamella-forming diblock copolymer nanoparticles. Specifically, microstructures with multiple spherical layers, that is, spherically concentric lamellae or onion-like structures, have been predicted in lamella-forming diblock copolymer nanoparticles by Monte Carlo (MC) simulations ${ }^{21,22}$ and by dynamic density functional theory simulations, ${ }^{23}$ and have been observed in the experiments with various methods. ${ }^{24-29}$ These studies demonstrate that the spherical boundaries of nanoparticles, especially those with preferential surfaces, can strongly influence the microstructures of diblock nanoparticles by distorting the flat lamellae into spherical ones. Moreover, the perpendicular lamellae, that is, the stacked lamellae, have been observed for diblock copolymer nanoparticles in experiments ${ }^{25,26,29,30}$ and simulations, ${ }^{22}$ in which the lamellae maintain the same flat characteristics as the bulk structures (perpendicular to one of symmetric axe). In addition to spherical and perpendicular lamellae, a series of non-lamellar microstructures, including the embedded structure and Janus-type, tennis ball-, mushroom- and screw-like structures, have also been observed in symmetric diblock copolymer nanoparticles. ${ }^{22,30}$ On the other hand, novel microstructures have been observed in cylinder-forming diblock nanoparticles by dissipative particle dynamics, ${ }^{31}$ dynamic density functional theory ${ }^{23}$ and self-consistent field theory (SCFT) approaches. ${ }^{32}$ The microstructures predicted in these simulations revealed that cylinder-forming diblock copolymers can be packed into complex microstructures with either body-centered symmetry or other asymmetries. However, there are relatively few studies that have contributed to the microstructures of cylinder-forming diblock copolymer nanoparticles.

Although the studies mentioned above have been carried out with individual confinement sizes and strengths of surface fields, it is still noteworthy that only a few works involve confinement size- and surface-field-induced effects. ${ }^{22,23,30-32}$ In particular, Yu et al. ${ }^{22}$ have

${ }^{1}$ Department of Physics, Wenzhou University, Wenzhou, Zhejiang, China and ${ }^{2}$ CAS Key Laboratory of Soft Matter Chemistry, University of Science and Technology of China, Hefei, Anhui, China

Correspondence: Dr S Li and Dr Y Ji, Department of Physics, Wenzhou University, Wenzhou, Zhejiang 325035, China.

E-mail: shibenli@wzu.edu.cn and yyji@wzu.edu.cn

Received 29 January 2011; accepted 10 March 2011; published online 27 April 2011 
investigated the confinement-induced effects on the lamella-forming diblock copolymers in nanoparticles. These authors have examined the dependence of spherical layer thicknesses and chain conformations on confinement size and surface fields, and have revealed a rich variety of novel structures in diblock nanoparticles. Chen et al. ${ }^{32}$ have reported confinement size effects on cylinder-forming diblock nanoparticles, and have observed multilayered microstructures with bodycentered symmetries. In these two studies, the model copolymers were adopted to be located in the strong segregation regime. Generally speaking, the microstructures of diblock copolymer nanoparticles are strongly dependent on the polymer parameters, that is, the block ratios and the degrees of incompatibility. ${ }^{23,29}$ We are aware that the polymer parameters near the phase-diagram boundaries are of special interest because they are able to deform into a series of microstructures for the diblock copolymers under confinement. ${ }^{13}$ Hence, an extensive study that examines the surface-field effects of the microstructures of cylinder-forming diblock copolymer nanoparticles, especially those with special polymer parameters, is desirable.

In this study, we systematically investigated the effects of surface fields on the microstructures of cylinder-forming diblock copolymer nanoparticles with the SCFT calculation method. The polymer parameters were selected to be near the cylinder-sphere phase boundaries, in which the bulk cylinders are easy to deform into the other structures. The novel structures in such polymer nanoparticles over a wide parameter space were explored.

\section{EXPERIMENTAL PROCEDURE}

We used the SCFT calculation method to obtain the microstructures of equilibrium states in diblock copolymer nanoparticles. The model is shown in Figure 1. The spherical boundary of a nanoparticle was constructed as an impenetrable surface; the diblock copolymers inside were regarded as Gaussian chains with the same volume fraction of A-blocks, $f_{\mathrm{A}}$, and polymerization index, $N$. In our calculations, the spatial lengths were scaled in the unit of $R_{\mathrm{g}}$, the gyration radius of an ideal Gaussian chain for a diblock copolymer; the free energies are expressed in units of $k_{B}$ T. For a diblock copolymer confined in a spherical nanoparticle with diameter $d$ and volume $V$, the SCFT free energy per chain in an external field can be expressed as

$$
\frac{F}{n k_{\mathrm{B}} T}=-\ln \left(\frac{Q}{V}\right)+\frac{1}{V} \int d r\left[\chi_{\mathrm{AB}} N \phi_{\mathrm{A}} \phi_{\mathrm{B}}-\omega_{\mathrm{A}} \phi_{\mathrm{A}}-\omega_{\mathrm{B}} \phi_{\mathrm{B}}-U_{\mathrm{A}} \phi_{\mathrm{A}}-U_{\mathrm{B}} \phi_{\mathrm{B}}\right]
$$

Equation (1) is divided into two terms, the logarithmic and integral terms. The integral term describes the interactions among the different blocks and those between the blocks and spherical confinement boundaries. The repulsion interactions between A and B blocks are parameterized by the incompatibility degree of the different blocks, $\chi N$, where $\chi$ is the Flory-Huggins parameter. This repulsion interaction is proportional to the respective segment density $\phi_{\mathrm{A}(\mathrm{B})}$. In SCFT, the fluctuation effects are ignored, and the multi-body interactions between the surrounding blocks are replaced by a mean field $\omega_{\mathrm{A}(\mathrm{B})}$. The adsorption interactions between the spherical boundaries and blocks, $U_{\mathrm{A}(\mathrm{B})}$, which are reduced to $\lambda_{\mathrm{A}(\mathrm{B})}=U_{\mathrm{A}(\mathrm{B})} / \chi N$, are only applied on the lattice next to the nanoparticle boundaries such that they are short-range interactions. This adsorption interaction is similar to that commonly used in MC simulations, which provides a convenient way to compare the present results with those from the available MC simulations. ${ }^{8,9,12,22}$ In the logarithmic term, the singlechain partition function, $Q$, can be expressed as $Q=\int q(r, 1) d \mathrm{r}$ in the mean fields $\omega_{\mathrm{A}(\mathrm{B})}$. The segment distribution function, $q(\mathbf{r}, s)$, is the statistical weight of a chain segment of contour length $s$ containing a free-chain end with its connected end located at $\mathbf{r}$. The function $q(\mathbf{r}, s)$ satisfies the modified diffusion equation in the mean fields; that is,

$$
\frac{\partial}{\partial s} q(\mathbf{r}, s)=R_{\mathrm{g}}^{2} \nabla^{2} q(\mathbf{r}, s)-N \omega q(\mathbf{r}, s)
$$

Because the chain is asymmetric, another segment distribution function, $q^{\prime}(\mathbf{r}, s)$, is needed, which satisfies the diffusion equation multiplied by -1 only on the right-hand side. These two equations assume the initial conditions $q(\mathbf{r}, 0)=q^{\prime}$ $(\mathbf{r}, 1)=1$. Here, $\omega=\omega_{\mathrm{A}}$ for $0 \leqslant s \leqslant f_{\mathrm{A}}$; otherwise, $\omega=\omega_{\mathrm{B}}$. Also, the CrankNicholson scheme is used to solve the modified diffusion equations, which have been proven to be an efficient approach in previous works. ${ }^{13,32}$

To obtain the equilibrium phase structure, the free energy is minimized to a stable value with respect to the mean fields and the segment density fields. The minimization of the free energy results in a set of self-consistent equations, which can be expressed as

$$
\begin{aligned}
& \omega_{\mathrm{A}}(\mathbf{r})=\chi\left(\phi_{\mathrm{B}}(\mathbf{r})-f_{\mathrm{B}}\right)-U_{\mathrm{A}}(\mathbf{r}) \\
& \omega_{B}(\mathbf{r})=\chi\left(\phi_{\mathrm{A}}(\mathbf{r})-f_{\mathrm{A}}\right)-U_{\mathrm{B}}(\mathbf{r}) \\
& \phi_{\mathrm{A}}(\mathbf{r})=\frac{V}{Q} \int_{0}^{f_{\mathrm{A}}} d s q(\mathbf{r}, s) q^{\prime}(\mathbf{r}, s) \\
& \phi_{\mathrm{B}}(\mathbf{r})=\frac{V}{Q} \int_{f_{\mathrm{A}}}^{1} d s q(\mathbf{r}, s) q^{\prime}(\mathbf{r}, s)
\end{aligned}
$$

where the incompressibility

$$
\phi_{0}(\mathbf{r})=\phi_{\mathrm{A}}(\mathbf{r})+\phi_{\mathrm{B}}(\mathbf{r})
$$

is enforced with a Lagrange multiplier $P(\mathbf{r})$. We generalize the incompressibility constraint to $\phi_{0}=\phi_{\mathrm{A}}+\phi_{\mathrm{B}}$ and $\phi_{0}=1$ inside the nanoparticle, $\phi_{0}=0.5$ in the lattice near the boundary and $\phi_{0}=0$ in the boundary. In other SCFT calculations, the incompressibility constraint is set to be a step function or a cosine function. ${ }^{33,34}$ Recently, Meng and Wang ${ }^{35}$ suggested that the microstructures of the equilibrium states are not significantly affected by the functional form for the incompressibility constraints. Therefore, these generalizations in our calculations ensure the representation of an impenetrable boundary and cause the diblock copolymers to be confined inside the spherical nanoparticles. The above self-consistent equations can be numerically solved by a combinatorial screening method, based on the real-space implementation originally proposed by Fredrickson and Drolet. ${ }^{36,37}$ The real-space implementation with the iteration steps is suitable for exploring the novel phase structures of copolymers without requiring assumptions of system symmetry. Here, we use the real-space implementation to determine the phases with minimum free energies. We use different random initial conditions in our iterative algorithm to determine the potential structures, and we take the equilibrium phase to be the structure with

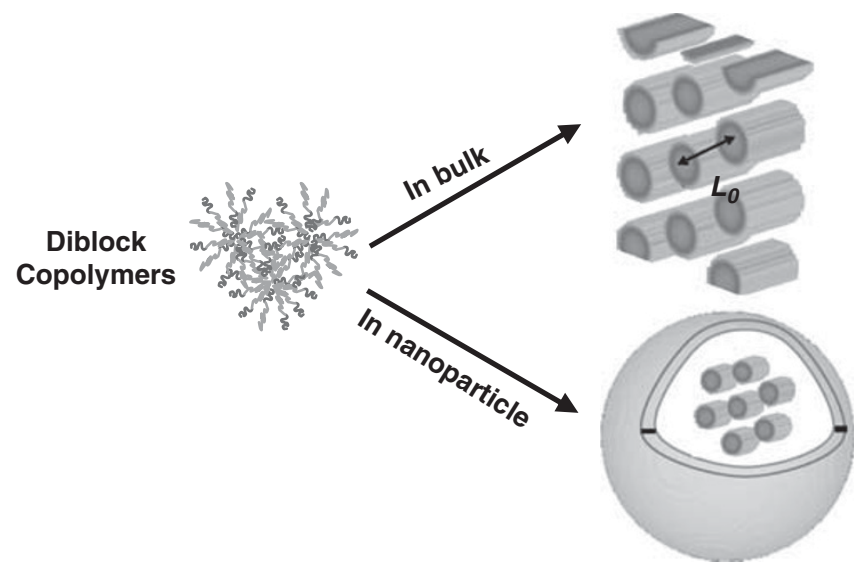

Figure 1 Schematic illustration of nanoparticles composed by A-B diblock copolymers, which are cylinder forming with a bulk period $L_{0}$. The red chains of the diblock copolymers are A-blocks, and the green chains are B-blocks. A full color version of this figure is available at Polymer Journal online. 
the lowest free energy when the convergence criterion is $<1.0 \times 10^{-10}$. In these calculations, we assigned $0.2 R_{g}$ as the lattice constant, and we divided the chain contour length into 200 segments, which is similar to what was performed in our previous works. ${ }^{13,38}$

In this study, we specified the polymer parameters as $f_{\mathrm{A}}=0.25$ and $\chi N=30$, which results in hexagonally packed cylinders in the bulk phase. According to the bulk phase diagram, the model polymer is located near the boundaries between the spherical and cylindrical phases. ${ }^{39}$ To obtain the bulk period of model polymers, we performed the SCFT calculations many times in the twodimensional space with periodic boundary conditions. Under these considerations, we obtained a bulk polymer period of $L_{0}=3.80 R_{g}$, as shown in Figure 1 . For the microstructures in spherical nanoparticles, several particle diameters $d$ were considered, and the strengths of the surface fields $\lambda_{\mathrm{A}(\mathrm{B})}$ were incrementally varied to explore the surface-field-induced effects on the microstructures of nanoparticles. All of these SCFT calculations were performed in a PC cluster with 25 crunodes.

\section{RESULTS AND DISCUSSION}

The surface-field-induced microstructures are shown in Figures 2-5, and more detailed information of several microstructures is also provided in the Supplementary information (see Supplementary Figures S1 and S2). We examined the surface-field-induced effects by increasing $\lambda_{\mathrm{A}(\mathrm{B})}$ with a small step of $\Delta \lambda_{\mathrm{A}(\mathrm{B})}=1 / 15$ over a wide range of $\lambda_{\mathrm{A}}=0.00-1.00$. The free energies as functions of surface-field strengths are plotted in Figure 6. All of these microstructures are presented by two colors (red and blue), which represent the A-blockrich and B-block-rich domains, respectively. We chose the surfacefield-induced effects only for the nanoparticles with $d / L_{0}=1.68,2.84$, 3.26 and 4.32 as typical examples; we denoted $d / L_{0}=1.68$ and 4.32 as the small and large nanoparticles, respectively, and $d / L_{0}=2.84$ and 3.26 as the moderate nanoparticles.

The microstructures in nanoparticles with A-preferential surface As shown in Figure 2, for small nanoparticles with $d / L_{0}=1.68$ (that is, severe confinement), the nanoparticles maintain their single-layered structures over the whole range. In MC studies, the symmetric diblock copolymers also keep the stacked lamellar structure over a wide range of surface-field strengths. ${ }^{22}$ Here, the polymer-surface interactions are similar to the contact interactions used in the MC simulations. Hence, it is difficult for the weak contact interactions to affect the singlelayered microstructures, especially for the contact interactions with the minority blocks. We then turned our attention to the microstructures of large nanoparticles at $d / L_{0}=4.32$, the structures with three layers, as shown in Figure 2. The same three-layered structure, as that in the neutral surface, appears over a wide range of $\lambda_{\mathrm{A}}=0.00-0.53$, which indicates that this structure is stable under this condition. With increasing $\lambda_{\mathrm{A}}$, the structure first turns into a structure with a cylinder network at $\lambda_{\mathrm{A}}=0.60-0.73$, and then the cylinder network develops into a spherical lamella at $\lambda_{\mathrm{A}}=0.80-1.00$. During this process, the distribution of A-blocks becomes more and more crowded in the outermost layer because of the strong adsorptions. The previous simulations reported that the lamella- or even cylinder-forming diblock copolymers can be adsorbed into the boundaries and assemble into cylindrical lamellae near the boundaries because of strong adsorption effects. ${ }^{15,16}$ It has also been reported that symmetric diblock copolymers form spherical lamellae near the boundaries because of the strong surface field. ${ }^{22}$ Here, we observed spherically concentric lamellae for cylinder-forming lamellae originating from the strong adsorption effects. From an overview of the evolutionary process, we conclude that the spherical surface field results in the corresponding symmetric structure, from body-centered symmetry to spherical symmetry, when increasing the strength of the surface field. Because

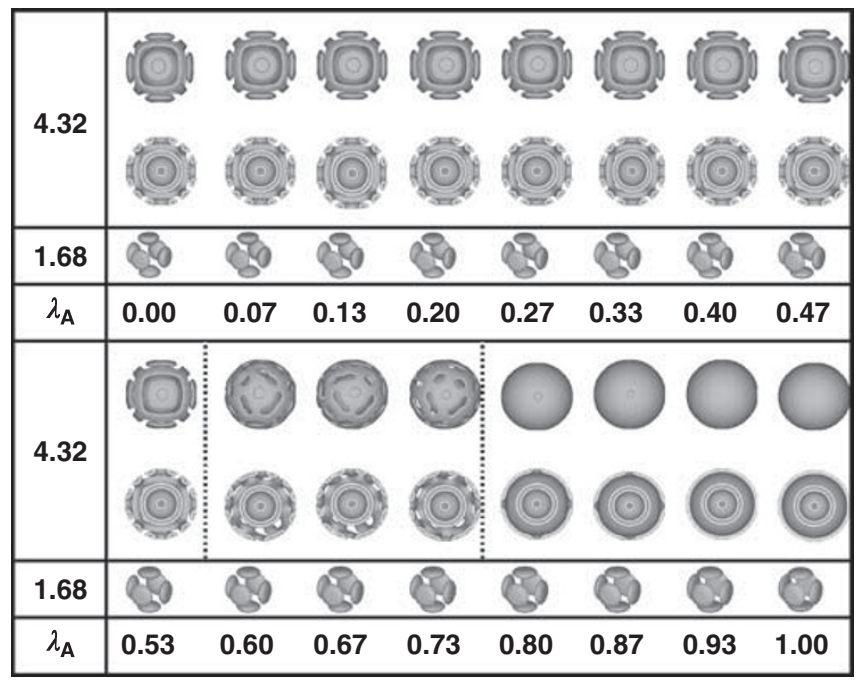

Figure 2 The microstructures of nanoparticles with $d / L_{0}=1.68$ and 4.32. The structures are arranged as a function of the strength of surface preference, $\lambda_{\mathrm{A}}$, in the nanoparticle with A-preferential surfaces. The full structures and their cut views are presented by A-blocks. A full color version of this figure is available at Polymer Journal online.

the long-range interactions between the middle layer and outermost layer are not sufficiently strong, the innermost and middle layers remain unchanged. An additional reason may be that the innermost and middle layers possess the original spherical symmetries, which is consistent with the symmetries of the surface fields.

For moderate nanoparticles with double-layered structures, we selected two cases, the $d / L_{0}=2.84$ and 3.26 cases, in which the inner layers are solid spheres and spherical shells in the neutral surface, to examine the surface-field effects, as shown in Figure 3. For $d / L_{0}=2.84$, the rhombic-cylinder network only occurs in the narrow range of $\lambda_{\mathrm{A}}=0.00-0.07$ and then evolves into a structure with irregular distorted cylinders in the outer layer and an ellipsoid in the inner layer at $\lambda_{\mathrm{A}}=0.13-0.20$. The ellipsoid structure with weak symmetry is assumed to be accommodated with the distorted cylinders in the outer layer through the long-range interactions between the inner and outer layers to balance the short-range polymer-surface interactions applied on the outer layers. In turn, this balance results in the irregular distribution of cylinders in the outer layer. We observed other irregular structures in the outer layers, such as when $d / L_{0}=4.32$ and $\lambda_{\mathrm{A}}=0.60$ 0.73 , in which the weak asymmetries in the outer layers enabled us to observe these accommodation effects. It should be pointed out that the long-range interactions between the neighboring periods likely lead to artifact microstructures in the bulk simulations when the periodic boundary conditions are selected. ${ }^{40,41}$ In our calculations, the periodic boundary conditions are not necessary because the nanoparticle provides a completely closed confinement. Alternatively, this requires the distortion of cylinders in the outer layer to meet commensurability. Subsequently, the outer layer adjusts its own pattern into a structure with six cylinder rings in the outer layer at $\lambda_{\mathrm{A}}=0.27-0.67$. However, the ellipsoid structure in the inner layer undergoes a long process of adjustment and evolves into a solid sphere. This fact indicates that the short-range interactions between polymers and the surfaces have more obvious effects than those of long-range interactions between neighboring cylinders. The strong surface field requires the spherical symmetries applied on the outer layers, and six cylinder rings then deform to connect with each other and develop into network structures with weak spherical symmetry at 


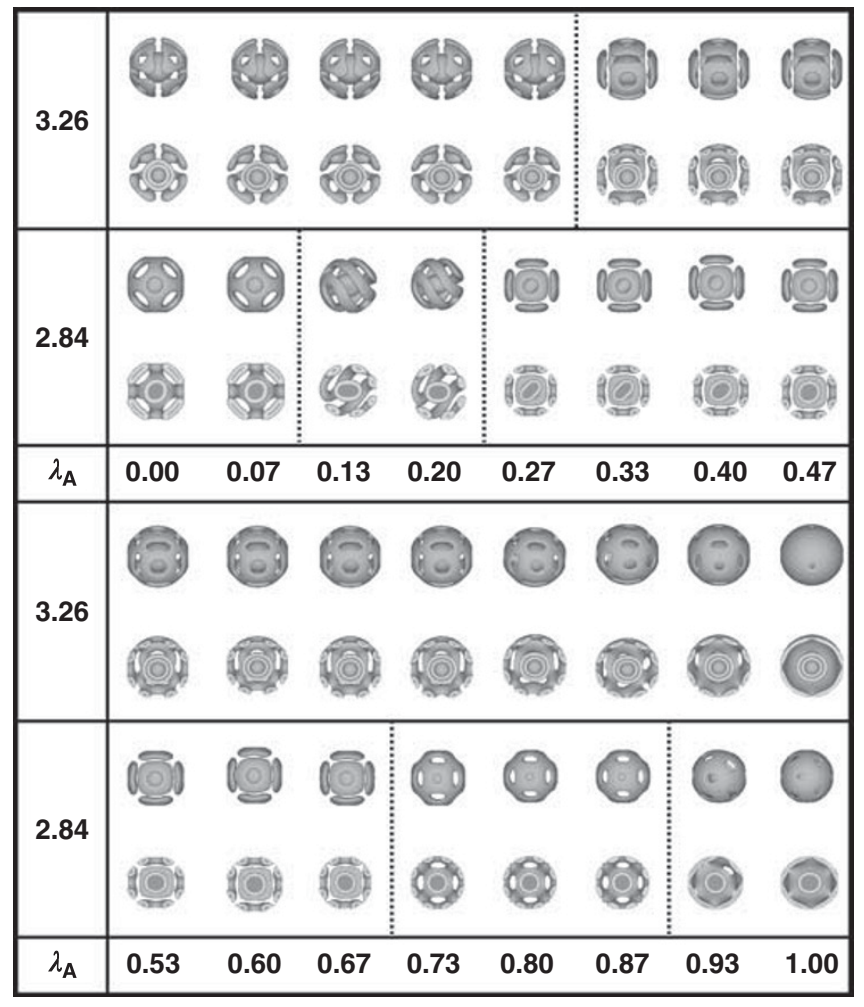

Figure 3 The microstructures of nanoparticles with $d / L_{0}=2.84$ and 3.26. The structures are arranged as a function of the strength of surface preference, $\lambda_{A}$, in the nanoparticle with A-preferential surfaces. The full structures and their cut views are presented by A-blocks. A full color version of this figure is available at Polymer Journal online.

$\lambda_{\mathrm{A}}=0.73-0.87$ and subsequently into a spherical lamella structure at $\lambda_{A}=0.93-1.00$. In this process, the variation of the outer layer does not affect the inner structure, although the short-range polymer-surface interactions are strong. For another double-layered structure at $d / L_{0}=3.26$, only three structures were observed during the evolution process with $\lambda_{\mathrm{A}}$. First, the diblock copolymers maintain a structure with eight cylinder triangles and spherical shells over a range of $\lambda_{\mathrm{A}}=0.00-0.27$, indicating that this structure is not sensitive to the weak $\lambda_{\mathrm{A}}$. Then, the structure with six cylinder rings and a spherical shell appears at $\lambda_{\mathrm{A}}=0.33-0.47$, which is similar to the structures at $d / L_{0}=2.84$ and $\lambda_{A}=0.40-0.67$. With increasing $\lambda_{\mathrm{A}}$, the outer layer continuously adjusts its pattern and finally evolves into a spherical lamella at $\lambda_{\mathrm{A}}=1.00$.

Here, we emphasize two characteristics by comparing the surfacefield effects on the nanoparticles with various layer structures. First, the appearance of spherical lamellae has a delay of $\lambda_{\mathrm{A}}$ for the first time as the confinement size increases. Specifically, we observed the spherical lamellae at $\lambda_{\mathrm{A}}=0.80$ for the three-layered structure and at $\lambda_{A}=1.00$ for the double-layered structure; we were unable to observe the spherical lamellae in the range of $\lambda_{\mathrm{A}}=0.00-1.00$ for the single-layer structure. Indeed, spherically concentric lamellae have been reported for lamellae-forming diblock copolymers under spherical confinement in MC simulations ${ }^{21,22}$ and in experiments. ${ }^{24,25}$ More evidence has been provided in the cylindrical nanopore cases, in which the cylinderforming diblock copolymers form the cylindrical lamellae when the confinement sizes and strengths of the surface field are increased. ${ }^{16}$ Here, we observed the phase behaviors of spherically concentric lamellae for the cylinder-forming diblock copolymer nanoparticles due to strong polymer-surface interactions. On the other hand, the

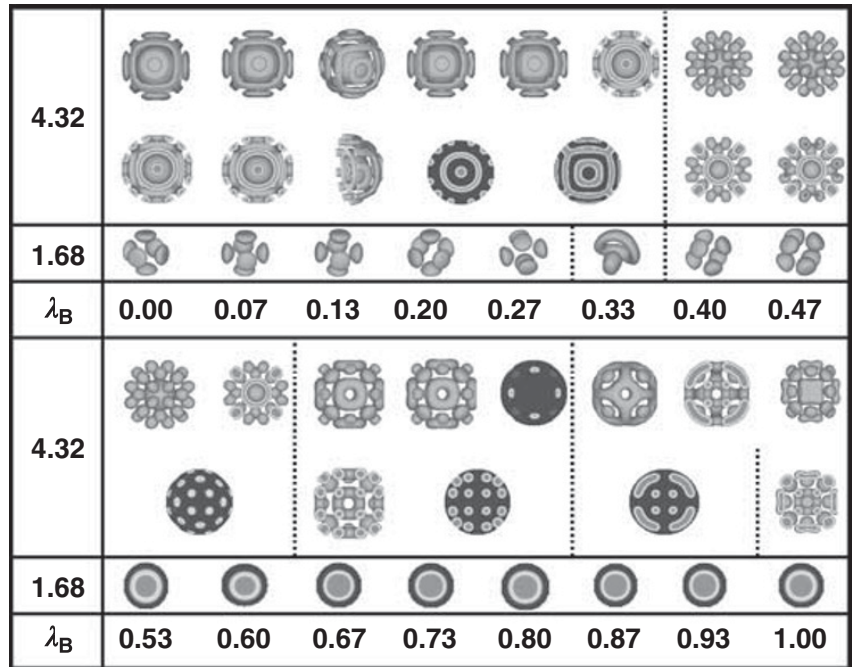

Figure 4 The microstructures of nanoparticles with $d / L_{0}=1.68$ and 4.32 . The structures are arranged as a function of the strength of surface preference, $\lambda_{B}$, in the nanoparticle with B-preferential surfaces. The full structures and their cut views are presented by A-blocks. A full color version of this figure is available at Polymer Journal online.

balance between the long-range and short-range interactions is dependent on the nanoparticle diameters. The results suggest that the short-range polymer-surface interactions dominate in the large nanoparticles, whereas the long-range interactions between the inner and outer layers have more important roles in the small nanoparticles. This also suggests that studies should be conducted on the stress distributions for cylinder-forming diblock copolymers in nanoparticles, which has been recently developed for bulk diblock copolymers in theoretical and MC simulation works. ${ }^{42,43}$

The microstructures in nanoparticles with B-preferential surface The strongly asymmetric blocks of model polymers enable us to compare the different effects of surface fields on the majority (B) and minority (A) blocks. Figures 4 and 5 provide us another case to examine the surface-field-induced effects on the microstructures of cylinder-forming diblock copolymer nanoparticles. For the small nanoparticles with single-layered structures at $d / L_{0}=1.68$, as shown in Figure 4, the surface field is not strong enough to influence the microstructures at $\lambda_{\mathrm{B}}=0.00-0.27$, such that the diblock copolymers maintain the same patterns as those in the neutral surfaces. Subsequently, the diblock copolymers form two structures, a structure with a ring and droplet at $\lambda_{\mathrm{B}}=0.33$ and a structure with several droplets at $\lambda_{\mathrm{B}}=0.40-0.47$; these then assemble into structures with spherical lamellae over a wide range of $\lambda_{\mathrm{B}}=0.53-1.00$. Because the surfaces prefer the B-blocks, the outermost layers are constructed by B-block spherical lamellae. These spherical lamellae are formed because of the strong short-range adsorption interactions between the boundaries and B-blocks. Once the spherical lamellae are formed, they show a stable structure over a wide range. For the large nanoparticles with three-layered structures at $d / L_{0}=4.32$, we observed that the microstructures are not sensitive to the weak surface field at $\lambda_{\mathrm{B}}=0.00-0.33$, as shown in Figure 4. For visual aid, we also plotted the cut views for the A- and B-block domains in the below. With these aids, we indeed found that the patterns of these domains remained unchanged over this range. When the surface field is increased to $\lambda_{\mathrm{B}}=0.40-0.60$, an interesting structure appears in which the three-layered structures deform into double-layered structures in A-block domains. This 


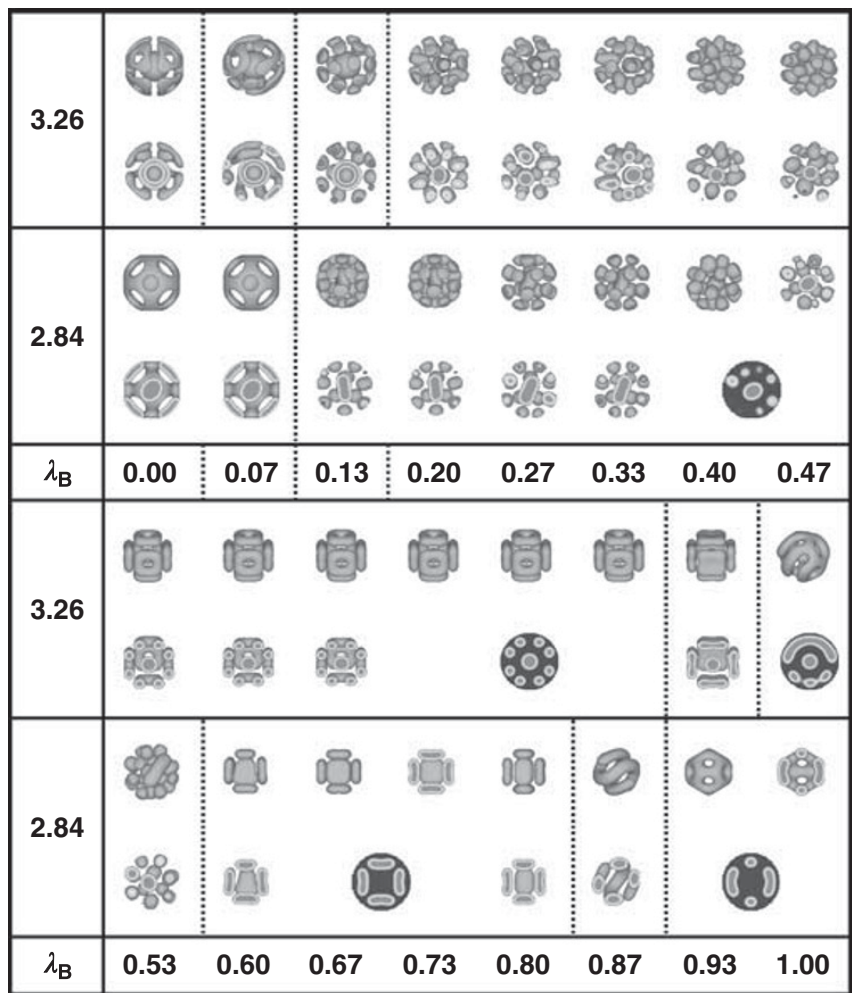

Figure 5 The microstructures of nanoparticles with $d / L_{0}=2.84$ and 3.26 . The structures are arranged as a function of the strength of surface preference, $\lambda_{B}$, in the nanoparticle with B-preferential surfaces. The full structures and their cut views are presented by A-blocks. A full color version of this figure is available at Polymer Journal online.

double-layered structure contains a large number of ellipsoids distributed spherically in the outer layer. A previous work has reported similar structures for a cylinder-forming diblock copolymer under spherical confinement with other parameters in which the inner layer is a solid sphere. ${ }^{32}$ Similar structures with scattered droplets have also been reported in other work. ${ }^{31}$ As the diblocks are highly asymmetric, the surface fields with $\lambda_{\mathrm{B}}=0.40-0.60$ are much stronger than those of A-preferential surfaces. To balance this strong adsorption interaction between the B-blocks and boundaries, the middle layer can easily be deformed, and the inner layer is enlarged from the solid core to a spherical shell. Following the same reasoning, the spherical shell is continuously deformed with increasing $\lambda_{\mathrm{B}}$ into a cubic cylinder at $\lambda_{\mathrm{B}}=0.67-0.80$. With continuously increasing $\lambda_{\mathrm{B}}$, the diblock copolymer nanoparticle transforms into a structure with rhombic cylinders embedded with droplets in the outer layers at $\lambda_{\mathrm{B}}=0.87-0.93$. Finally, the diblock copolymers deform into the droplets spherically distributed in the outer layer and a similar cylindrical cube in the inner layer at $\lambda_{\mathrm{B}}=1.00$. From the evolution process at $\lambda_{\mathrm{B}}=0.67-1.00$, we conclude that the cylindrical cube structure in the inner layers are relatively stable structures under the present conditions.

For the moderate nanoparticles with double-layered structures at $d / L_{0}=2.84$, shown in Figure 5, the diblock copolymers maintain their original patterns in the narrow range of $\lambda_{\mathrm{B}}=0.00-0.07$ and transform into another structure over a wide range of $\lambda_{B}=0.13-0.47$. The double-layered structure consists of droplets in the outer layers and an irregular solid core in the inner layers. The irregular core adjusts its pattern slightly with increasing $\lambda_{\mathrm{B}}$ and develops into a regular sphere at $\lambda_{\mathrm{B}}=0.40-0.47$. This slight adjustment in the inner layer is similar to those observed in the $d / L_{0}=2.84$ and $\lambda_{\mathrm{A}}=0.13-0.33$ case. We noticed

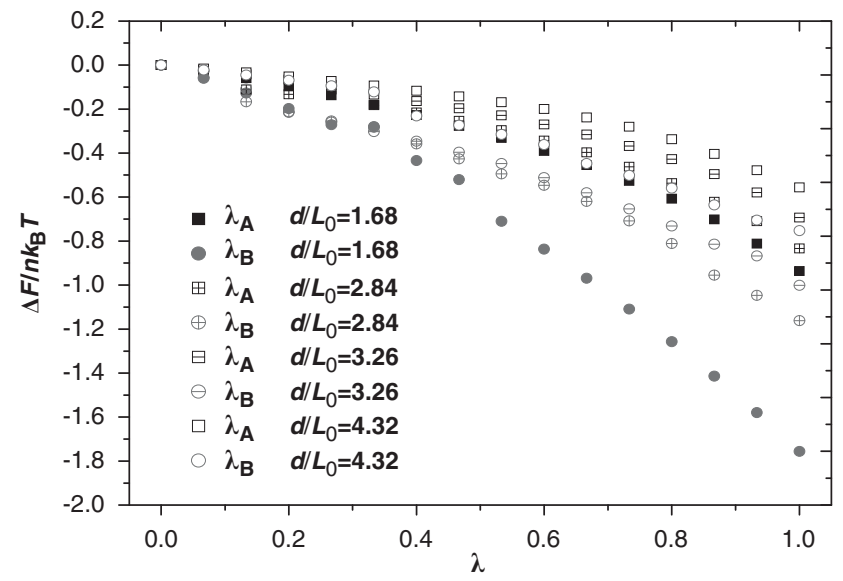

Figure 6 The free energies shift from the corresponding energies in neutral surfaces, $\Delta F / n k_{B} T=F / n k_{B} T-F_{0} / n k_{B} T$, as functions of surfacefield strengths $\lambda$ in nanoparticles with $A$ - and $B$-preferential surfaces for various confinement sizes of $d / L_{0}=1.68,2.84,3.26$ and 4.32 . A full color version of this figure is available at Polymer Journal online.

that this structure is similar to that reported in a previous work in which the blocks have a more asymmetric characteristic. ${ }^{23}$ In particular, we observed the several droplets connected with each other in the outer layers at $\lambda_{\mathrm{B}}=0.53$. The structure immediately transits into a single-layer structure, in which the diblock copolymers form the cubic layers in the B-block matrix at $\lambda_{\mathrm{B}}=0.60-80$. These cubic lamellae are somewhat similar to the cubic cylinders in the $\lambda_{\mathrm{A}}=0.40-0.67$ and $d / L_{0}=2.84$ case, in which the diblock copolymers form double-layered structures. The single-layer structure is due to the strong adsorption interactions between the surfaces and B-blocks, resulting in a spherical lamella near the boundaries. Then the structure with cubic symmetry evolves into a structure with body-centered symmetry at $\lambda_{\mathrm{B}}=0.93-$ 1.00 and passes through an immediate structure with distorted cylinders at $\lambda_{\mathrm{B}}=0.87$. The nanoparticles with $d / L_{0}=3.26$ experience a similar development process, in which the scattered droplets in the outer layer and solid sphere in the inner layers also appear at $\lambda_{B}=0.20$ 0.47 after a few adjustment steps at $\lambda_{\mathrm{B}}=0.07$ and 0.13 . Then the diblock copolymer shows the same structures as those observed at $d / L_{0}=2.84$ and $\lambda_{\mathrm{A}}=0.40-0.67$ over the wide range of $\lambda_{\mathrm{B}}=0.53-1.00$ in addition to the special structure at $\lambda_{\mathrm{B}}=1.00$.

The general conclusion that the strong adsorption interactions between the surfaces and B-blocks are equivalent to reducing the confinement sizes can be drawn. This equivalence is particularly obvious in the nanoparticles with double- or three-layered structures. Specifically, the strong interactions result in the transformation of the three-layered structures into the double-layered structures at $d / L_{0}=4.32$ and the double-layered structures into the single-layered structure at $d / L_{0}=3.26$ and 2.84. In addition, we point out that the microstructures are more complex than those observed in A-preferential surfaces because of the hard-wall effects in which the A-blocks prefer the neutral surfaces. ${ }^{35}$ These phenomena have also been observed for diblock copolymers with cylindrical nanopores. ${ }^{13,14,16}$ Hence, the short-range interactions between the B-blocks and boundaries must overcome these hard-wall effects by overturning the blocks near the boundaries, resulting in the redistribution of polymer chains through interactions between neighboring cylinders. At the same time, the long-range interactions between the inner and outer layers are modified to accommodate the surface-polymer interactions to balance these interactions. This is why many droplets are distributed in the 
outer layers, and the diblock copolymer nanoparticles have more complex structures when the B-preferential surface fields are applied on the nanoparticle boundaries. Unfortunately, the strength of surface field is usually unknown in experiments for the boundary of a nanoparticle, which hampers the precise comparison between the present results and other experiments. It is likely that the electrospinning technique that creates pore confinement may be extended to control the surface field of nanoparticles. ${ }^{44}$

\section{Structural transition between the microstructures}

To investigate the effects of surface fields on the microstructures in more detail, we plotted the free energies as functions of surface-field strengths in Figure 6. For convenient comparison, the free energies depart from the corresponding neutral surface cases. The free energies have a decreasing trend with increasing $\lambda$ because the energies of adsorption interactions are negative. The significant decrease with the strengths of the surface field appears in the small nanoparticle case, although there are relatively slight changes in the free energies of the large nanoparticles. Importantly, all of these free energy curves show that the phase transitions are of the first order, although there are tiny structural transitions in small nanoparticles with A-preferential surfaces. Previous works have reported that the confinement-sizeinduced phase transitions are generally considered of the first order in nanopores and thin films. ${ }^{16,45,46}$ Our previous work also suggests that the surface-field-induced phase transitions are of the first order for diblock copolymers confined in nanorod arrays. ${ }^{47}$ The present observations are in agreement with those results from cylindrical, thin-film confinements and nanorod array confinements. Another characteristic is that the decrease in the free energies of the B-preferential surfaces is more significant than that of the A-preferential surfaces, indicating that the appearance of complex structures at B-preferential surfaces is due to severe asymmetry between the two blocks in the confinement system. These significant changes in the structures may be explained by the hard-wall effects, as mentioned previously. Therefore, the surface needs more adsorption energy to counteract the adsorptions of A-blocks, and the interval adsorptions between the distinct blocks lead to significant variations in the self-assembled microstructures.

\section{Conclusion}

In this work, we carried out a SCFT study on the surface-field-induced microstructures of cylinder-forming diblock copolymer nanoparticles. The typically small, moderate and large nanoparticles with single, double and three layers have been considered, and a rich variety of novel microstructures have been predicted in cylinder-forming diblock copolymer nanoparticles with A- and B-preferential surfaces.

For the A-preferential surface, it is difficult to influence the small nanoparticles with single-layered structures and the spherical shells (rather than the outer layer) in the large nanoparticles with double- or three-layered structures because of them having the same spherical symmetry as those of the surface fields. Moreover, the nanoparticles tend to assemble into concentric spherical lamellae when the surface field is sufficiently strong. For the B-preferential surfaces, we observed that the nanoparticles with single-layered structures transform into spherical lamella structures because of the strong adsorption interactions between the boundaries and the B-blocks. The spherically concentric lamellae were not observed in the nanoparticles with double- or three-layered structures, although the surface field is sufficiently strong. Instead, the number of layers decreases in the B-preferential surface cases, as spherical lamellae form near the boundaries, which is equivalent to reducing the confinement size. The inner layer varies significantly as $\lambda_{\mathrm{B}}$ increases. For the surface- field-induced effects, it was suggested that the balance between the short-range surface-polymer interactions and the long-range cylinder interactions drive the double-layered structure into a relatively diverse evolution process, and the novel structures can be understood based on the accommodation between the inner and outer layers. On the other hand, investigation of the free energies indicated that the free energies decrease in the surface-field-induced cases, in which the more significant decreases appear in the nanoparticles with either small diameters or B-preferential surfaces. The variation in free energy also suggests that the surface-field-induced transitions are of the first order. The results in the present work are compared with the available experiments and simulations, which are in general agreement. Our observations are expected to not only enrich our knowledge of surface-field-induced microstructures in nanoparticles but also be helpful in the fabrication of diblock copolymer nanoparticles with controlled patterns.

\section{ACKNOWLEDGEMENTS}

This research was supported by the General Program of National Natural Science Foundation of China (Nos. 20774066, 20974081, 20934004, 50773072, 20974001 and 21074096), the Outstanding Youth Fund of China (No. 20525416), the National Basic Research Program of China (No. 2005CB623800) and the Natural Science Foundation of Zhejiang Province (Nos. Y4090174, Y6100033).

1 Otsuka, H., Nagasaki, Y. \& Kataoka, K. PEGylated nanoparticles for biological and pharmaceutical applications. Adv. Drug. Delivery Rev. 55, 403-419 (2003).

2 Branco, M. C. \& Schn, J. P. Self-assembling materials for therapeutic delivery. Acta Biomaterialia 5, 817-831 (2009).

3 Gourevich, I. L., Field, M., Wei, Z., Paquet, C., Petukhova, A., Alteheld, A., Kumacheva, E., Saarinen, J. J. \& Sipe, J. E. Polymer multilayer particles: a route to spherical dielectric resonators. Macromolecules 39, 1449 (2006).

4 Kakizawa, Y., Miyata, K., Furukawa, S. \& Kataoka, K. Size-controlled formation of a calcium phosphate-based organic-inorganic hybrid vector for gene delivery using poly(ethylene glycol)-block-poly(aspartic acid). Adv. Mater. 16, 699-702 (2004).

5 Pham, H. H., Gourevich, I., Oh, J. K., Jonkman, J. E. N. \& Kumacheva, E. A multidye nanostructured material for optical data storage and security data encryption. Adv. Mater. 16, 516-520 (2004).

6 Wu, Y., Cheng, G., Katsov, K., Sides, S., Wang, J., Tang, J., Fredrickson, G. H. Moskovits, M. G. \& Stusky, D. Composite mesostructures by nano-confinement. Nat. Mater. 3, 816-822 (2004).

7 Xiang, H., Shin, K., Kim, T., Moon, S. I., McCarthy, T. J. \& Russell, T. P. Block copolymers under cylindrical confinement. Macromolecules 37, 5660-5664 (2004).

8 Chen, P., He, X. \& Liang, H. Effect of surface field on the morphology of a symmetric diblock copolymer under cylindrical confinement. J. Chem. Phys. 124, 104906 (2006).

9 Feng, J. \& Ruckenstein, E. Morphology transitions of AB diblock copolymer melts confined in nanocylindrical tubes. J. Chem. Phys. 125, 164911 (2006).

10 Feng, J. \& Ruckenstein, E. Morphologies of AB diblock copolymer melts confined in nanocylindrical tubes. Macromolecules 39, 4899-4906 (2006).

$11 \mathrm{Li}$, W. \& Wickham, R. A. Self-assembled morphologies of a diblock copolymer melt confined in a cylindrical nanopore. Macromolecules 39, 8492-8498 (2006).

12 Yu, B., Sun, P., Chen, T., Jin, Q., Ding, D., Li, B. \& Shi, A.- C. Confinement-induced novel morphologies of block copolymers. Phys. Rev. Lett. 96, 138306 (2006).

13 Chen, P., Liang, H. \& Shi, A.- C. Origin of microstructures from confined asymmetric diblock copolymers. Macromolecules 40, 7329-7335(2007).

14 Yu, B., Sun, P., Chen, T., Jin, Q., Ding, D., Li, B. \& Shi, A- C. Self-assembly of diblock copolymers confined in cylindrical nanopores. J. Chem. Phys. 127, 114906 (2007).

15 Sevink, G. J. A. \& Zvelindovsky, A. V. Block copolymers confined in a nanopore: path finding in a curving and frustrating flatland. J. Chem. Phys. 128, 084901 (2008).

16 Yu, B., Jin, Q., Ding, D., Li, B. \& Shi, A.- C. Confinement-induced morphologies of cylinder-forming asymmetric diblock copolymers. Macromolecules 41, 4042-4054 (2008).

$17 \mathrm{Li}$, W. \& Wickham, R. A. Influence of the surface field on the self-assembly of a diblock copolymer melt confined in a cylindrical nanopore. Macromolecules 42, 7530-7536 (2009).

$18 \mathrm{Ma}$, M., Titievsky, K., Thomas, E. L. \& Rutledge, G. C. Continuous concentric lamellar block copolymer nanofibers with long range order. Nano Lett. 9, 1678-1683 (2009). 
19 Sohn, K. E., Kojio, K., Berry, B. C., Karim, A., Coffin, R. C., Bazan, G. C., Kramer, E. J., Sprung, M. \& Wang, J. Surface effects on the thin film morphology of block copolymers with bulk order-order transitions. Macromolecules 43, 3406-3414 (2010).

20 Jung, J., Park, H.- W., Lee, S., Lee, H., Chang, T., Matsunaga, K. \& Jinnai, H. Effect of film thickness on the phase behaviors of diblock copolymer thin film. ACS Nano 4, 3109-31016 (2010).

$21 \mathrm{He}$, X., Song, M., Liang, H. \& Pan, C. Self-assembly of the symmetric diblock copolymer in a confined state. J. Chem. Phys. 114, 10510-10513 (2001).

22 Yu, B., Li, B., Jin, Q., Ding, D. \& Shi, A- C. Self-assembly of symmetric diblock copolymers confined in spherical nanopores. Macromolecules 40, 9133-9142 (2007).

23 Fraaije, J.G.E.M., \& Sevink,, G. J. A. Model for pattern formation in polymer surfactant nanodroplets. Macromolecules 36, 7891-7893 (2003).

24 Arsenault, A. C., Rider, D. A., Ttreault, N., Chen, J. I.- L., Coombs, N., Ozin, G. A. \& Manners, I. Block copolymers under periodic, strong three-dimensional confinement. J.Am.Chem.Soc. 127, 9954-9955 (2005).

25 Yabu, H., Higuchi, T. \& Shimomura, M. Unique phase-separations of block copolymer nanoparticles. Adv. Mater. 17, 2062-2065 (2005).

26 Higuchi, T., Tajima, A., Yabu, H. \& Shimomura, M. Spontaneous formation of polymer nanoparticles with inner micro-phase separation structures. Soft Matter 4, 1302-1305 (2008).

27 Rider, D. A., Chen, J. I. L., Eloi, J.- C., Arsenault, A. C., Russell, T. P., Ozin, G. A. $\&$ Manners, I. Controlling the morphologies of organometallic block copolymers in the 3-dimensional spatial confinement of colloidal and inverse colloidal crystals. Macromolecules 41, 2250-2259 (2008).

28 Tanaka, T., Saito, N. \& Okubo, M. Control of layer thickness of onion-like multilayered composite polymer particles prepared by the solvent evaporation method. Macromolecules 42, 7423-7429 (2009).

29 Higuchi, T., Motoyoshi, K., Sugimori, H., Jinnai, H., Yabu, H. \& Shimomura, M. Phase transition and phase transformation in block copolymer nanoparticles. Macromol. Rapid.Commun. 31, 1773-1778 (2010).

30 Higuchi, T., Tajima, A., Motoyoshi, K., Yabu, H. \& Shimomura, M. Frustrated phases of block copolymers in nanoparticles. Angew. Chem. Int. Ed. 47, 8044-8046 (2008).

31 Feng, J., Liu, H. \& Hua, Y. Micro-phase separation of diblock copolymer in a nanosphere: dissipative particle dynamics approach. Fluid Phase Equilibria 261, 50-57 (2007).

32 Chen, P., Liang, H. \& Shi, A.- C. Microstructures of a cylinder-forming diblock copolymer under spherical confinement. Macromolecules 41, 8938-8943 (2008).
33 Matsen, M. W. Thin films of block copolymer. J. Chem. Phys. 106, 7781-7791 (1997).

34 Li, W., Wickham, R. A. \& Garbary, R. A. Phase diagram for a diblock copolymer melt under cylindrical confinement. Macromolecules 39, 806-811 (2006).

35 Meng, D. \& Wang, Q. Hard-surface effects in polymer self-consistent field calculations. J.Chem.Phys. 126, 234902 (2007).

36 Drolet, F. \& Fredrickson, G. H. Combinatorial screening of complex block copolymer assembly with self-consistent field theory. Phys. Rev. Lett. 83, 4317-4320 (1999).

37 Fredrickson, G. H., Ganesan, V. \& Drolet, F. Field-theoretic computer simulation methods for polymers and complex fluids. Macromolecules 35, 16-39 (2002).

$38 \mathrm{Li}$, S., Chen, P., Wang, X., Zhang, L. \& Liang, H. Surface-induced morphologies of lamella-forming diblock copolymers confined in the period arrays. J.Chem.Phys. 130, 014902 (2009).

39 Matsen, M. W. \& Schick, M. Stable and unstable phase of a diblock copolymer. Phys.Rev.Lett. 72, 2660-2663 (1994).

40 Wang, Q., Yan, Q., Nealey, P. F. \& de Pablo, J. J. Monte Carlo simulations of diblock copolymer thin films confined between two homogeneous surfaces. J. Chem. Phys. 112, 450-464 (2000).

41 Wang, Q., Nealey, P. F. \& de Pablo, J. J. Monte Carlo simulations of asymmetric diblock copolymer thin films confined between two homogeneous surfaces. Macromolecules 34, 3458-3470 (2001).

42 Maniadis, P., Lookman, T., Kober, E. M. \& Rasmussen, K. Ø. Stress distributions in diblock copolymers. Phys. Rev. Lett. 99, 048302 (2007).

43 Detcheverry, F. A., Pike, D. Q., Nealey, P. F., Muller, M. \& de Pablo, J. J. Monte Carlo simulation of coarse grain polymeric systems. Phys. Rev. Lett. 102, 197801 (2009).

$44 \mathrm{Ma}$, M., Krikorian, V., Yu, J. H., Thomas, E. L. \& Rutledge, G. C. Electrospun polymer nanofibers with internal periodic structure obtained by microphase separation of cylindrically confined block copolymers. Nano Lett. 6, 2969-2972 (2006).

45 Wang, Q. Symmetric diblock copolymers in nanopores: Monte Carlo simulations and strong-stretching theory. J. Chem. Phys. 126, 024903 (2007).

46 Geisinger, T., Muller, M. \& Binder, K. Symmetric diblock copolymers in thin films. I. Phase stability in self-consistent field calculations and Monte Carlo simulations. J. Chem. Phys. 111, 5241-5250 (1999).

47 Li, S., Ji, Y., Chen, P., Zhang, L. \& Liang, H. Surface-induced phase transitions in dense nanoparticle arrays of lamella-forming diblock copolymers. Polymer 51, 4994-5001 (2010).

Supplementary Information accompanies the paper on Polymer Journal website (http://www.nature.com/pj) 